\title{
A Novel Evolutionary Approach to Software Cost Estimation
}

\author{
Disha Guleria \\ Baddi University, Solan HP
}

\begin{abstract}
Human Opinion Dynamics is a novel approach to solve complex optimization problems. This paper proposes and implements Human Opinion Dynamics for tuning the parameters of COCOMO model for Software Cost Estimation. The input is coding size or lines of code and the output is effort in Person-Months. Mean Absolute Relative Error and Prediction are the two objectives considered for the fine tuning of parameters. The dataset considered is COCOMO. The current paper demonstrates that use of human opinion dynamics illustrated promising results. It has been observed that when compared with standard COCOMO it gives better results.
\end{abstract}

\section{Keywords}

Human Opinion Dynamics, COCOMO, MARE, Social influence, Update Rule

\section{INTRODUCTION}

When the information available is not complete or imperfect with less computation capacity and there is a need to find out the best solution from a large set of sample solutions then meta-heuristic or partial search algorithm is used to provide a solution to an optimization problem in Computer Science or mathematical optimization. There are various meta-heuristics available like, Multi-objective Particle Swarm Optimization [1], Genetic algorithm, Ant colony optimization [2], Bacteria Foraging Optimization, Genetic Algorithm, BAT Algorithm, Memetic Algorithm, and Firefly Algorithm etc. Extensive research has been done on these over large no. of applications. There is no exhaustive research using human opinion dynamics. Human opinion dynamics finds its applications in the areas of social physics. However recently a researcher throws light on the use of human opinion dynamics for solving complex mathematical optimization problems applied on some benchmark mathematical functions and compared the results with PSO[3].As Human being is the highest creature so the algorithm inspired by human creative problem solving process can be useful and provide better results. It is used as dynamic social impact theory as an optimized for the optimization of an impedance-tongue and results are compared with Genetic Algorithm and PSO[7].

\section{HUMAN OPINION DYNAMICS}

It is a meta-heuristic technique to solve complex optimization problem based upon human creative problem solving process. Understanding the concept of collective decision making, the study of opinion dynamics and opinion formation is important. It has been one of the most significant areas in social physics. Human Interactions give RISE to different kind of opinions in a society[3].In social network the formation of different kinds of opinions is an evolutionary process. There are several models describing human interaction networks like cultural dynamics, opinion dynamics, crowd behavior, human dynamics etc utilised for search strategies and complex mathematical optimization problems. The process of collective intelligence from the tendencies of social influence with effects of individualization escapade for developing search strategies [3]. The algorithm formed based upon the opinion formation structure of individuals. The algorithm is governed by four basic essential elements: Social Structure, Opinion Space, Social influence, Updating Rule.

\subsection{Social Structure}

The Interaction between individuals, group of individuals, frequency of interaction and the way they interacts comes under social structure. There are number of models like Cellular automata, Small world, Random graphs etc have been proposed and simulated in social physics [1].The models are explained below:

\subsubsection{Cellular Automata Model}

This model was first considered by Von Neumann studied in 1950 as a model used in biological systems. It works according to some set of rules based on the states of neighboring cells. It is affected by the presence of corresponding or adjacent neighbor. It is a discrete model used in complex science and biological processes. An example of cellular automata model is given below in table 1 which is having a system of cell objects exhibiting the following characteristics as follows:

- $\quad$ All the cells live on Grid

- Each cell has a state which might have two possibilities 0 or 1 or referred as yes or no.

- Neighborhood of cell can be defined in plenty of ways but most probably the list of adjacent cells.

- The figure represents grid of "cells" each "yes" or "no" and the colored part presents the neighborhood of cells.

Table 1. Example of Cellular Automata Model

\begin{tabular}{|l|l|l|l|l|l|l|}
\hline Yes & No & Yes & No & No & Yes & No \\
\hline No & Yes & Yes & Yes & No & No & Yes \\
\hline No & Yes & No & No & Yes & Yes & No \\
\hline Yes & Yes & No & Yes & No & No & Yes \\
\hline No & No & Yes & No & Yes & Yes & No \\
\hline Yes & Yes & No & Yes & No & No & Yes \\
\hline
\end{tabular}




\begin{tabular}{|l|l|l|l|l|l|l|}
\hline No & No & Yes & No & Yes & Yes & No \\
\hline No & Yes & Yes & Yes & No & No & Yes \\
\hline
\end{tabular}

\subsubsection{Random Graph}

Random graph can be well defined as a random process of probability distribution over graphs. The application area of random graph in the areas where complex networks need to be designed.

\subsubsection{Small World}

It is the type of network where most nodes are not influenced by the neighbors but finding ways to reach each other by small number of steps.

\subsection{Social Influence}

It is the influence of individuals on each other and they act according to others actions or suggestions. "Equation (1) describes social influence $u_{i j}(t)$ of individual $j$ on individual $i$ is given by

$u_{i j}(t)=\frac{S R_{j}(t)}{d_{i j}(t)}$

Where $d_{i j}(\mathrm{t})$ is the Euclidean distance between individuals .Social Ranking (SR) is based upon the fitness value of individuals.

\subsection{Updating Rule}

This rule is used to update the position of individuals in the search space .As it is dynamic in nature so change of position according to the best fitness value needs to be updated. In context to optimization problems it determines the new updated position of individuals. Equation (2) demonstrates the formula for updating rule

$\Delta O_{i}=\frac{\sum_{j=1}^{N}\left(O_{j}(t)-O_{i}(t) u_{i j}(t)\right.}{\sum_{j=1}^{N} u_{i j}(t)}+\epsilon_{i}(t), j \neq 1(2)$

Where $\Delta O_{i}$ represents updating rule, $o_{j}(\mathrm{t})$ is the opinion of number of individuals, $u_{i j}(\mathrm{t})$ represents Social influence, $\epsilon_{i}(t)$ is a normally distributed random noise with mean zero and $\mathrm{N}$ is the number of neighbours.

\subsection{Opinion Space}

There are two types of opinions of the individuals continuous or discrete. Continuous is the one which takes real values. Discrete takes values in the given range $[0,1]$ or $[1,-1]$.

\section{HUMAN OPINION DYNAMICSIN SOFTWARE COST ESTIMATION}

Cost Estimation is an important activity and can be done throughout the entire life cycle of the software product to be developed. It is the process of calculation of effort used for the development of project. Time and budget are the two important factors in software project management. The main focus is on time and budget in software project development [4].There are various models used for the effort calculation in software cost estimation. One of the most widely used algorithmic model is COCOMO .The parameters of COCOMO tuned with the help of meta-heuristic techniques. In this paper Human Opinion Dynamics is used to optimize the parameters of COCOMO

\subsection{COCOMO}

COCOMO model is developed by Boehm and have been widely used for the calculation of effort. Effort calculated by COCOMO model is measured in terms of size and constant value parameters a,b,c. using Intermediate COCOMO II model in which effort can be calculated using equation (3). "Equation (3) gives the formula for effort".

$$
\text { Effort }=a *(\text { size })^{b} * E A F+c(3)
$$

where size is the size of project measured in LOC(lines of code) or KLOC.EAF are effort multipliers. The value of parameters $a=3 b=1.2$.As these values are fixed for COCOMO model but these parameters vary from organization to organization depends on various factors like environmental factors. So, there is a need to tune the value of parameters to obtain better result in terms of accuracy and less error.

\subsection{Fitness function}

Fitness function is that function which is used to evaluate that which opinion is performing best and gives best results.

Each objective has some weight which is used to combine the two objectives into single objective. The weights assigned must be equal to one.

$$
\mathrm{W} 1+\mathrm{W} 2=1
$$

Hence, using the fitness function where the need is to minimize the error and maximize the prediction. Most Real World problems involves optimization of two or more objectives [1].A multi objective optimization function involve minimization of one and maximization of other. The fitness function used in our equation is based upon two objectives MARE i.e. mean absolute relative error and prediction.

"Equation (4) defines the fitness function used in the said approach."

$$
\text { function }=w 1(M A R E)+w 2(1-\text { pred. })(5)
$$

Where MARE is mean absolute relative error and Prediction (n) is the project having n\% error."Equation (6) gives the formula to calculate mean absolute error"

$$
\% M A R E=\sum\left[\frac{\text { abs(mes.effort }- \text { est.effort })}{(\text { mes.effort })}\right] / n(6)
$$

Where ' $n$ ' is the total number of projects and Mes. Effort is the actual measured effort and est. Effort is the effort calculated by using cost model COCOMO II and proposed technique. 


\section{PROPOSED METHODOLOGY}

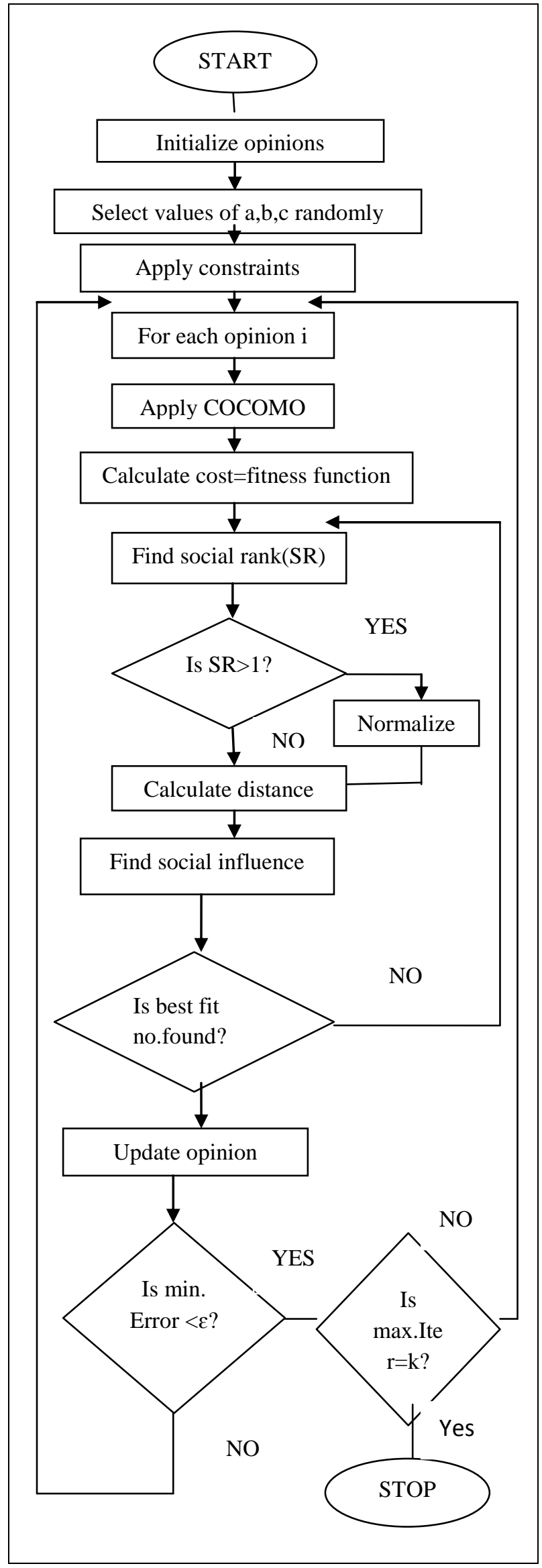

- $\quad$ Step 1: START

- Step 2: Initialize the opinions from 1 to 30

- Step 3:Assign any random value to $a, b, c$

- $\quad$ Step 4:Apply constraints (range of a, b, c)

- Step 5:For every value of opinion $i$ of each individual.

- $\quad$ Step6:Apply COCOMO formula and calculate effort with the calculated values of parameters a,b,c.

- Step 7:For these values of a,b,c, store the value of fitness function as the cost function which is to be minimized

- $\quad$ Step 8:Find Social Rank for the present value or opinion

- $\quad$ Step 9:If Social rank is greater than 1then normalize and then calculate distance i.e. Euclidean distance between two individuals.

- $\quad$ Step 10:Find social influence of the opinion which is based upon social rank and Euclidean distance

- Step 11: If best fit solution is found i.e, the opinion which fits aptly in the fitness function then go to Step 12 otherwise go to step 7 .

- Step 12: Update the value of Opinion

- Step 13: If minimum error $<\varepsilon$ (minm error) and maximum iteration $=\mathrm{k}$ then stop,otherwise go to Step 5 and perform the optimization process again.

\section{PERFORMANCE RESULTS AND IMPLEMENTATIONS}

This section describes the experimentation part. For testing the effectiveness of proposed models, it is tested on COCOMO dataset. Two datasets of 20 projects and 21 projects are considered. Tuned value of parameters obtained by implementing the above methodology.

Experiment 1.Total 20 projects are considered from COCOMO dataset . Total no. of iterations performed $=100$, no. of opinions $=30$. The optimized values of $a, b, c$ obtained are $\mathrm{a}=4.2, \mathrm{~b}=1, \mathrm{c}=1.3$.

1) The table given below is showing the values that are already available in dataset from COCOMO dataset .

TABLE 2. Experimental results for the comparison of effort

\begin{tabular}{|r|r|r|} 
Meas COCOMO Effo HOD Effort \\
\hline 240 & 347.2294197 & 224.744 \\
\hline 33 & 55.15808369 & 43.052 \\
\hline 8 & 12.18428797 & 10.292 \\
\hline 79 & 115.5563114 & 84.38 \\
\hline 9 & 11.53574316 & 12.9044 \\
\hline 7.3 & 7.475113353 & 7.688 \\
\hline 5.9 & 6.196705766 & 6.26756 \\
\hline 47 & 70.20610386 & 47.252 \\
\hline 8 & 10.44854672 & 8.8556 \\
\hline 8 & 8.648095925 & 8.78 \\
\hline 6 & 5.548713573 & 4.265 \\
\hline 45 & 66.75800317 & 50.297 \\
\hline 36 & 49.08832967 & 35.408 \\
\hline 41 & 71.19532527 & 64.136 \\
\hline 14 & 25.52408244 & 24.299 \\
\hline 20 & 16.51001299 & 15.8864 \\
\hline 70 & 100.7635967 & 75.434 \\
\hline 50 & 73.60772685 & 51.62 \\
\hline 38 & 48.82798088 & 15.08 \\
\hline 15 & 18.54325035 & \\
\hline & & 42.653 \\
\hline
\end{tabular}


2) Table 3 given below represents the actual effort and compared to effort calculated by COCOMO and proposed method.

TABLE 3 Results for Comparison of error
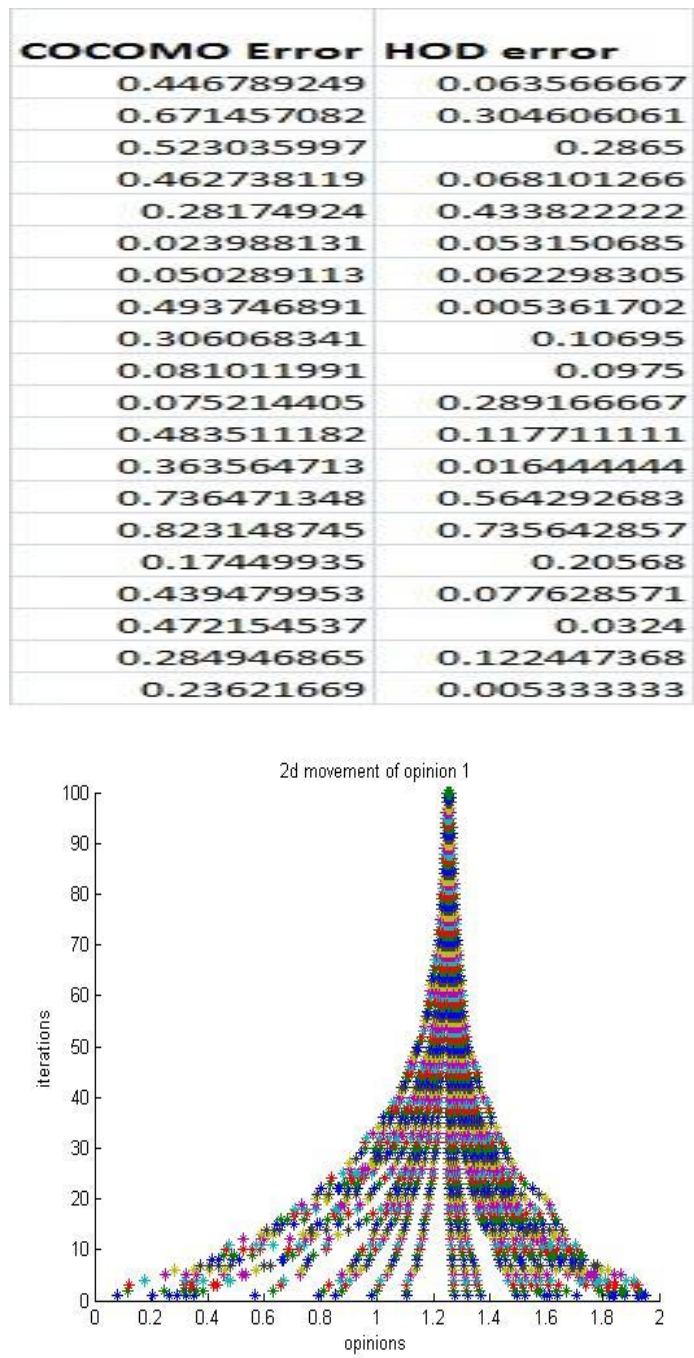

Fig. 1.Represents $2 \mathrm{~d}$ movement of convergence of opinions for opinion 1

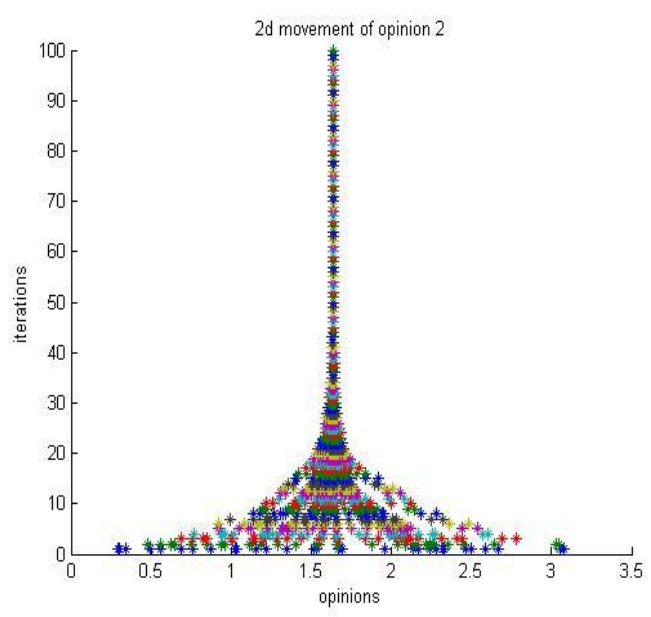

Fig..2 .Represents the plot of convergence of opinions for opinion 2

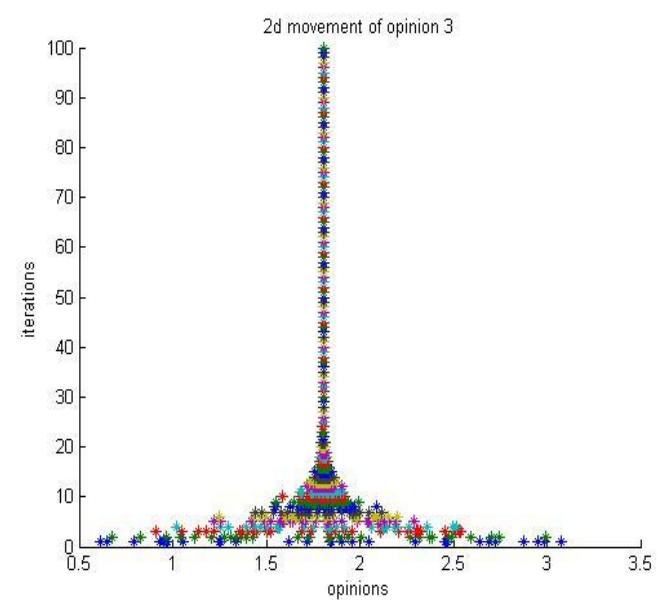

Fig.3.Represents the 2d movement of convergence of opinion

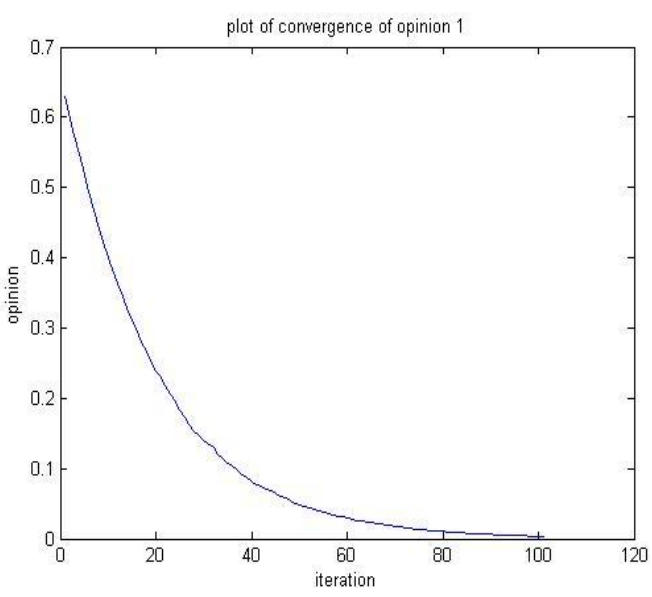

Fig.4 .Represents the plot of convergence of opinion 1 when 100 iterations are performed.

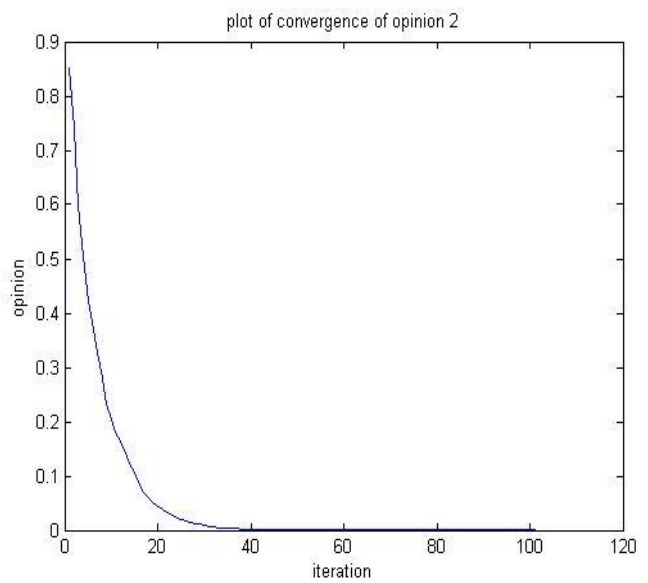

Fig..5. Represents the plot of convergence for opinion 


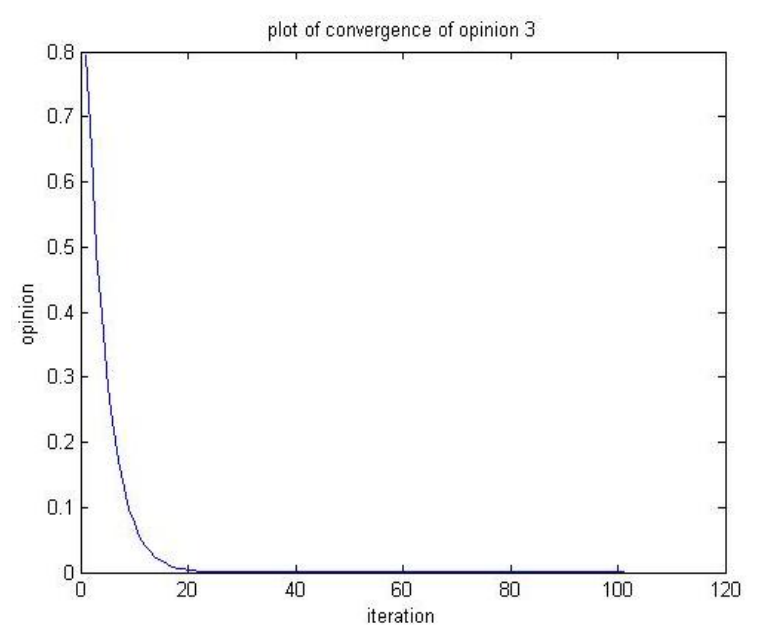

Fig .6.Represents the plot for convergence of opinion 3

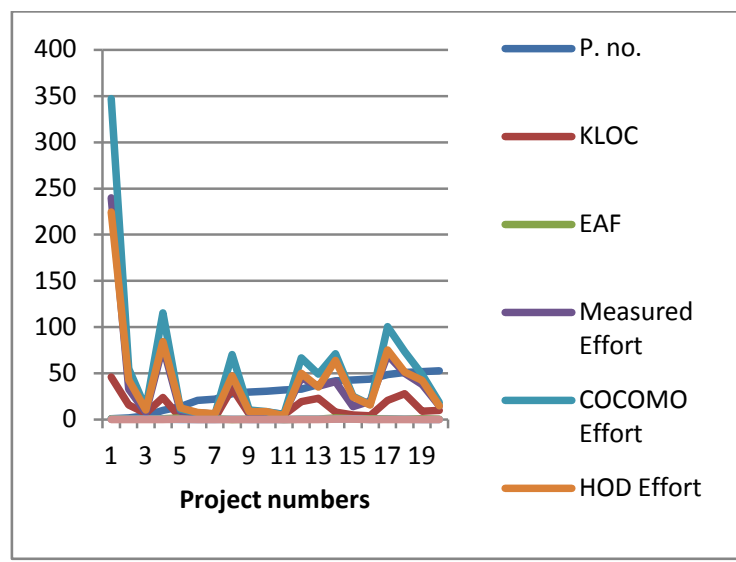

Fig .7.Represents the dataset values which consists of project number, lines of code, effort multipliers (EAF) and measured effort.

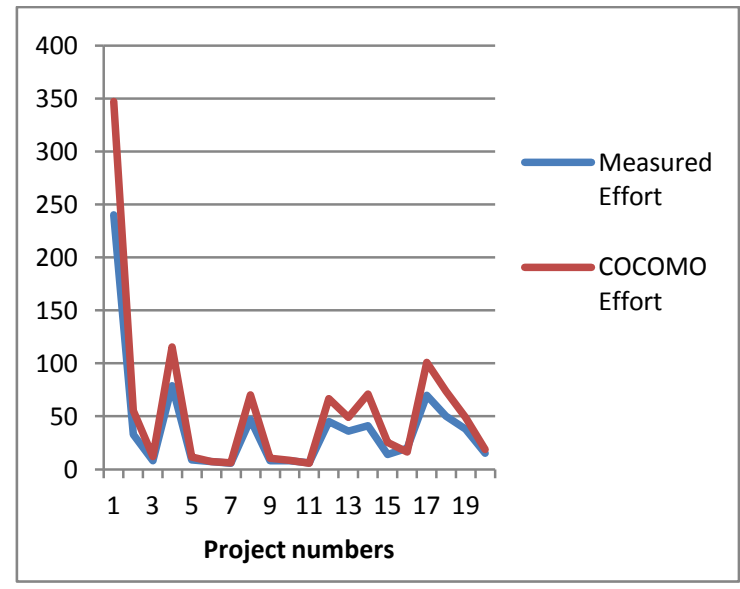

Fig. 8. Represents the comparison of COCOMO effort with measured effort

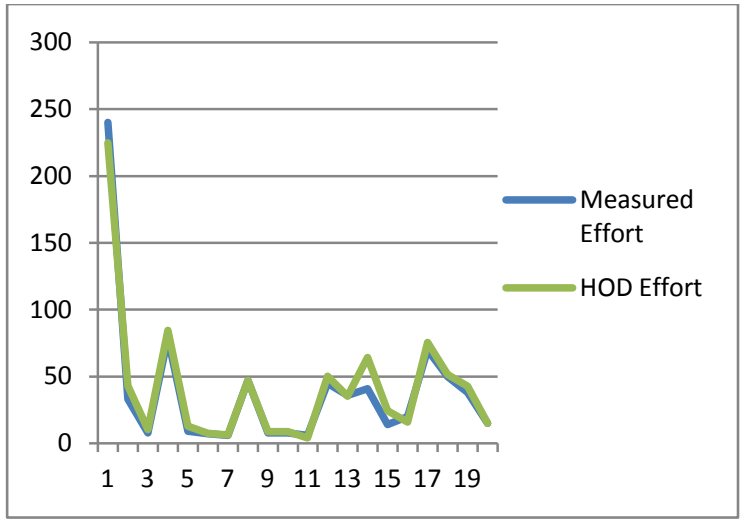

Fig..9. Represents the actual measured effort and effort calculated by human opinion dynamics

Effort calculated by Human Opinion Dynamics is more close to the actual measured effort. Thus our proposed approach gives better results.

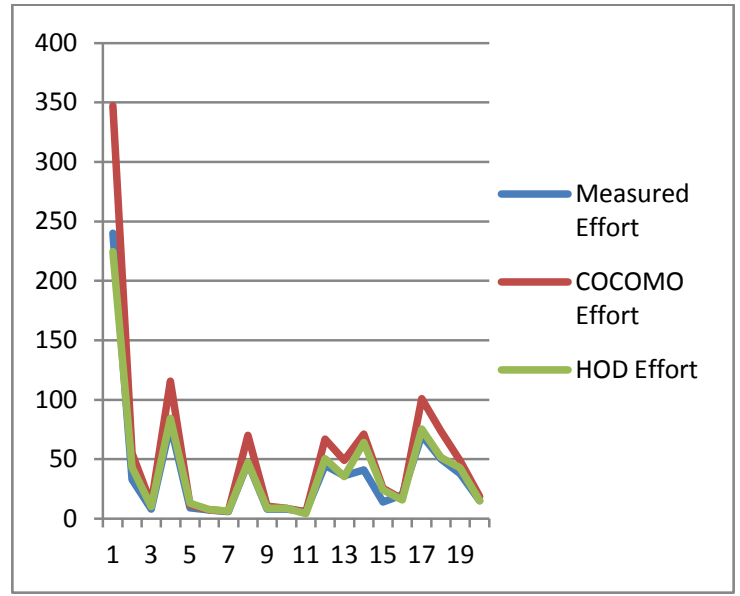

Fig.10.Respresents the comparison between actual effort, COCOMO effort and effort calculated by Human Opinion Dynamics

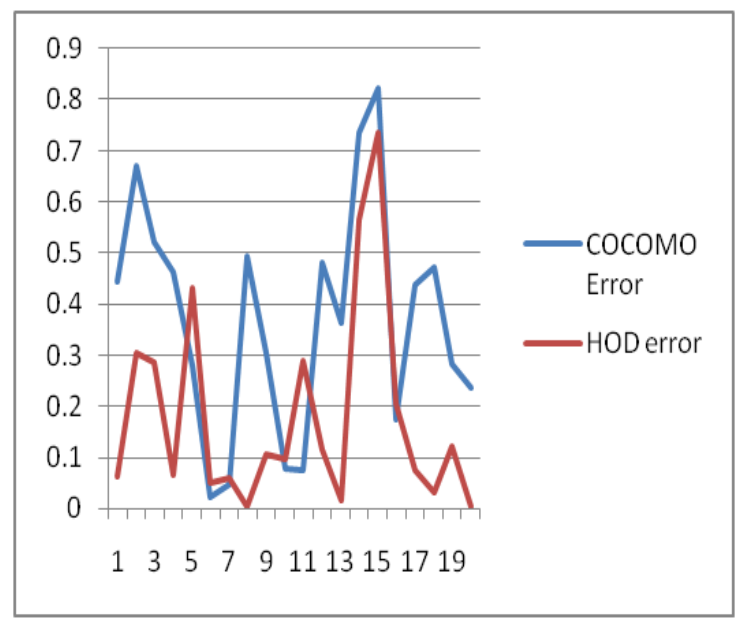

Fig.11. Represents the mean absolute relative error between COCOMO and human opinion dynamics, thus the error of COCOMO is more than the error of human opinion dynamics. 
COCOMO error is more than the error calculated by human opinion dynamics.

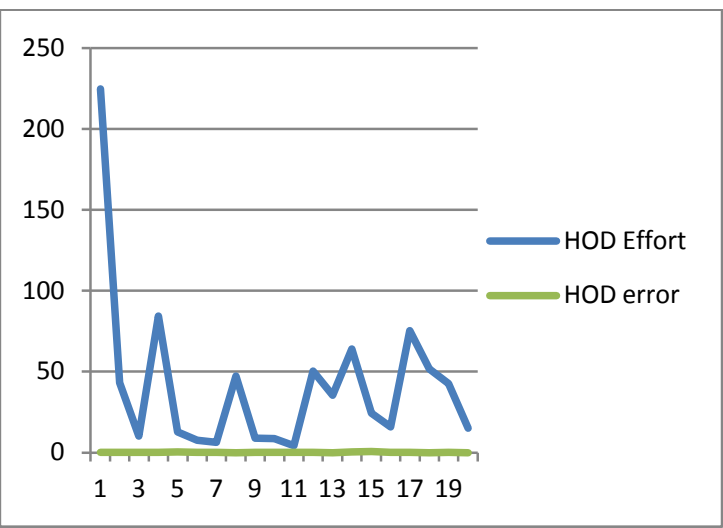

Fig .12.Represents calculated effort and error of human opinion dynamics

Experiment 2. Total 21 projects are considered from COCOMO dataset for testing the model. The tuned value of parameters obtained $\mathrm{a}=3.9, \mathrm{~b}=1.1, \mathrm{c}=5.8$.

Table 4. Represent the results of comparison of effort calculated by COCOMO, measured and human opinion dynamics.

TABLE 4. Experimental results of comparison of effort

\begin{tabular}{|r|r|r|}
\hline Meas & COCOMO Effo HOD Effort \\
\hline 240 & 347.2294197 & 302.010963 \\
\hline 33 & 55.15808369 & 48.5426138 \\
\hline 43 & 35.15169074 & 33.98172143 \\
\hline 8 & 12.18428797 & 7.257456815 \\
\hline 107 & 933.2187223 & 884.803766 \\
\hline 423 & 424.6827895 & 387.1125386 \\
\hline 321 & 229.1000679 & 217.2700783 \\
\hline 218 & 259.9765682 & 244.6808678 \\
\hline 201 & 255.9654486 & 226.1020171 \\
\hline 79 & 115.5563114 & 103.5240969 \\
\hline 73 & 65.69984976 & 70.72363706 \\
\hline 61 & 55.75777155 & 57.46190997 \\
\hline 40 & 40.5200193 & 40.4160499 \\
\hline 9 & 11.53574316 & 8.264152613 \\
\hline 539 & 474.8809821 & 395.0870279 \\
\hline 453 & 464.8472928 & 379.52697 \\
\hline 523 & 460.1465625 & 409.9776829 \\
\hline 387 & 362.3003293 & 314.0069941 \\
\hline 88 & 90.05438122 & 87.769738 \\
\hline 7.3 & 7.475113353 & 3.205747324 \\
\hline & & 178.3232425 \\
\hline
\end{tabular}

\section{CONCLUSION AND FUTURE SCOPE}

The Software Cost Estimation problem was dealt with in this paper which is a very important problem in the SDLC cycle as it influences the decision making process. A novel algorithms known as Human Opinion Dynamics based Optimization to estimate the Software Cost using intermediate COCOMO-II model. The software costs are predicted and the results are found to be quite better than that of the normal COCOMO-II model. The results when compared among HOD and COCOMO that the HOD's performance is quite better than the COCOMO's results both in terms of convergence and accuracy.

In future, other meta-heuristics algorithms can be applied and the results can be compared with our proposed methodology. The advanced COCOMO models can also be utilized and the approach can be utilized to develop an online system which would automatically predict the software cost serving as an automated feedback system for the business analyst.

\section{REFERENCES}

[1] Rao, G. Sivanageswara, Ch V. PhaniKrishna, and K. RajasekharaRao., "Multi Objective Particle Swarm Optimization for Software Cost Estimation", In ICT and Critical Infrastructure: Proceedings of the 48th Annual Convention of Computer Society of India-Vol I, pp. 125132. ,Springer International Publishing, Year 2014.

[2] Maleki, Isa, Ali Ghaffari, and Mohammad Masdari. ,"A new approach for software cost estimation with hybrid genetic algorithm and ant colony optimization," International Journal of Innovation and Applied Studies 5, no. 1,pp. 72-81, Year (2014).

[3] Kaur, Rishemjit, Ritesh Kumar, Amol P. Bhondekar, and PawanKapur.,"Human opinion dynamics: An inspiration to solve complex optimization problems."Scientific reports 3, Year (2013).

[4] Bardsiri, VahidKhatibi, DayangNorhayatiAbangJawawi, SitiZaitonMohdHashim, and ElhamKhatibi. "A PSObased model to increase the accuracy of software development effort estimation." Software Quality Journal 21, no. 3 ,pp 501-526, Year (2013).

[5] Benala, TirimulaRao, KoradaChinnababu, Rajib Mall, and SatchidanandaDehuri, "A Particle Swarm Optimized Functional Link Artificial Neural Network (PSOFLANN) in Software Cost Estimation," In Proceedings of the International Conference on Frontiers of Intelligent Computing: Theory and Applications (FICTA), pp. 59-66. Springer Berlin Heidelberg, Year (2013).

[6] Sheta, Alaa F., and Sultan Aljahdali. "Software effort estimation inspired by COCOMO and FP models: A fuzzy logic approach." International Journal of Advanced Computer Science and Applications (IJACSA) 4,pp no. 11, Year (2013).

[7] Bhondekar, Amol P., RishemjitKaur, Ritesh Kumar, RenuVig, and PawanKapur, "A novel approach using Dynamic Social Impact Theory for optimization ofimpedance-Tongue (iTongue)." Chemometrics and Intelligent Laboratory Systems 109, no. 1 ,pp: $65-$ 76, Year (2011). 